\title{
Demonstration of High-speed Indoor Optical Wireless Communications using Few-mode Based Uniform Beam Shaping
}

\author{
Jianghao Li \\ Department of Electrical and \\ Electronic Engineering \\ The University of Melbourne \\ Melbourne, VIC 3010, Australia \\ jianghaol1@student.unimelb.edu.au \\ Nicholas O'Keefe \\ Department of Electrical and \\ Electronic Engineering \\ The University of Melbourne \\ Melbourne, VIC 3010, Australia \\ nokeefe1@student.unimelb.edu.au
}

\author{
Christina Lim \\ Department of Electrical and \\ Electronic Engineering \\ The University of Melbourne \\ Melbourne, VIC 3010, Australia \\ chrislim@unimelb.edu.au \\ Ka-Lun Lee \\ Department of Electrical and \\ Electronic Engineering \\ The University of Melbourne \\ Melbourne, VIC 3010, Australia \\ alanl@unimelb.edu.au
}

\author{
Ampalavanapillai Nirmalathas \\ Department of Electrical and \\ Electronic Engineering \\ The University of Melbourne \\ Melbourne, VIC 3010, Australia \\ nirmalat@unimelb.edu.au
}

\begin{abstract}
A high-speed indoor optical wireless communications link using few-mode based uniform beam shaping is experimentally demonstrated. Results show that high-quality uniform beam can be realized and the BER performance can be improved significantly by combining the signals from different channels supported by different modes.
\end{abstract}

Keywords-optical wireless communications, few-mode fiber, uniform beam shaping, mode diversity

\section{INTRODUCTION}

In recent years, indoor optical wireless communications (OWC) has been extensively investigated as one of the promising solutions to meet the increasing demand for broadband communications, as the radio frequency based wireless access begins to suffer from capacity bottleneck [1], [2].

However, the light source which is widely used for OWC today is lasers producing narrow Gaussian-shaped beams with intensity distributions across the beam being not uniform. This makes the OWC systems more vulnerable to the link outage [3] depending on the location of the user with respect to users. More importantly, for multiuser OWC systems, different users can't share the same performance because of the non-uniform power distribution within the same cell. Therefore, the OWC systems with uniform power distribution lasers are highly needed. Many methods have been proposed to overcome this problem. One promising method is direct uniform beam shaping that transform the optical beam with a flat top or uniform intensity distribution directly. The most immediate method to achieve this goal is using diffractive or refractive optics (including lens, phase masks or lens arrays) to change the Gaussian shaped beam into a uniform shaped beam [4]. The other is based on indirect beam shaping which first splits the input beam into a lot of beamlets and then superposes them using beam homogenizers or integrators [5].

Both the diffractive or refractive optics and beam integrator techniques need to use lots of bulk optical elements that significantly sacrifice the system compactness and flexibility. Moreover, these two techniques need rigorous alignments and complicated operation. In this paper, for the first time, uniform beam shaping scheme by superposing few different spatial modes (few-mode) for indoor OWC systems was proposed and demonstrated. $10 \mathrm{~Gb} / \mathrm{s}$ on-off keying

This work was supported by Australian Research Council Discovery Project DP170100268.
(OOK) signals were transmitted over the uniform beam. The experimental results show high-quality uniformity (variation $\leq 4.1 \%$ across the beam) can be generated and the best bit error rate (BER) performance can be achieved by combining the signals from different channels of different modes using maximum ratio combining (MRC) scheme.

\section{EXPERIMENTAL SETUP FOR INDOOR OWC USING FEW-MODE UNIFORM BEAM SHAPING}

Uniform beam cannot be generated directly as it's not one of the eigenmodes supported by any optical fibers. However, it can be considered as a superposition of different shapes of modes, for example a fundamental mode along with a hollow ring-shaped mode [6], [7]. Fig. 1 describes the principle of how to generate the uniform beam. The uniform beam is shaped by superposing a donut-shaped LP $_{11}$ mode and $\mathrm{LP}_{01}$ mode incoherently, where the donut-shaped $\mathrm{LP}_{11}$ mode is obtained by adjusting the polarization states of the $\mathrm{LP}_{11}$ mode using polarization controls (PCs). Then, the data can be transmitted with the uniform beam as shown as the experimental setup in Fig.2. At the transmitter side, $10 \mathrm{~Gb} / \mathrm{s}$ OOK data was generated by an arbitrary waveform generator (AWG) and amplified by 2 electrical amplifiers (EAs). Amplified signals were used to drive two Mach-Zhender modulators (MZMs) to generate the OOK formatted data onto two optical signals from two lasers (LDs) with wavelengths of $1449 \mathrm{~nm}$ and $1550 \mathrm{~nm}$, respectively. Here, two different wavelengths were employed to generate incoherent addition of powers of $\mathrm{LP}_{01}$ and $\mathrm{LP}_{11}$ mode.

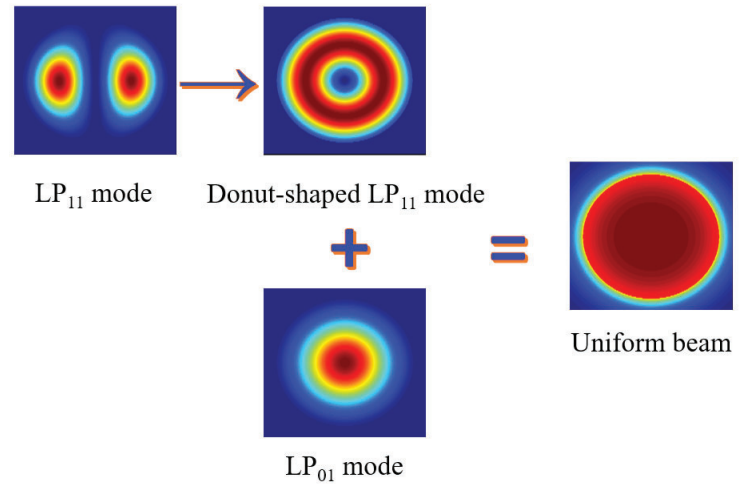

Fig. 1. Principle of few-mode uniform beam shaping. 


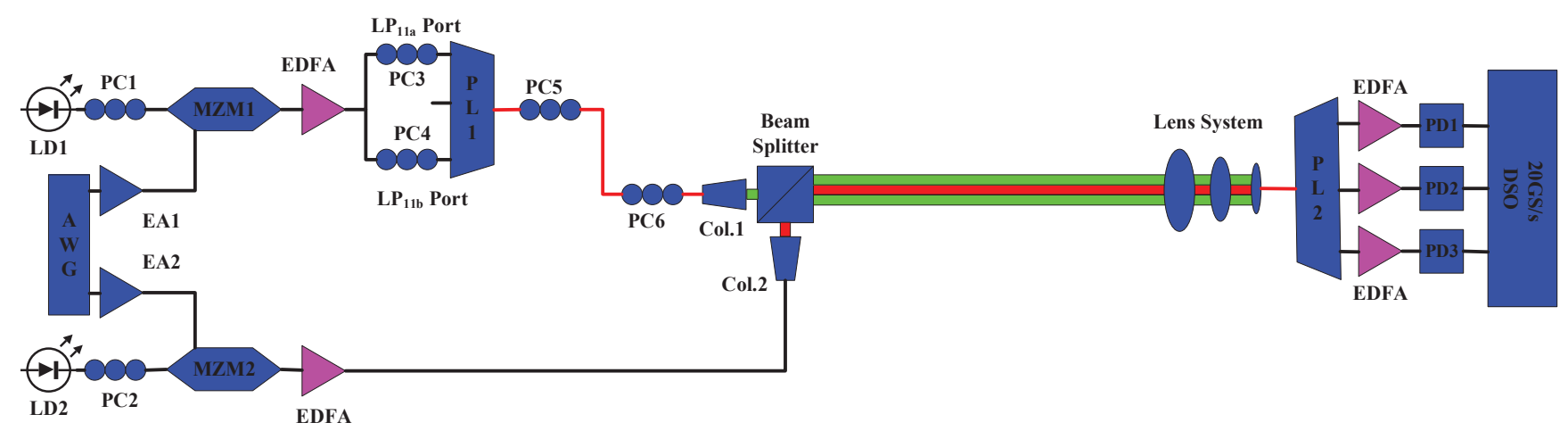

Fig. 2. Experimental setup.

$\mathrm{LP}_{11 \mathrm{a}}$ and $\mathrm{LP}_{11 \mathrm{~b}}$ modes were generated by the photonic lantern (PL) mode multiplexer after being amplified by an erbium-doped fiber amplifier (EDFA). Resulting signals were converted into a donut-shaped beam by adjusting PC36 , where PC5 and PC6 were entwined with few-mode fibers (FMFs) to control the polarization of $\mathrm{LP}_{11}$ modes. After that, the donut beam and the $\mathrm{LP}_{01}$ beam were superposed by a 50:50 beam splitter to get a uniform beam. In the experiment, the optical power at the output of the beam splitter was $4.5 \mathrm{dBm}$ which satisfies the limitation of the eye and skin safety [8].

At the receiver, the optical signals were collected by a lens system composed of 3 lenses with different size and focal lengths and coupled into the few-mode FMF after $1.2 \mathrm{~m}$ indoor free-space transmission. Then a photonic lantern can demultiplex the few-mode signals into 3 branches of $\mathrm{LP}_{01}$ mode signals. The 3 branches of signals were amplified by 3 EDFAs and then detected by $310 \mathrm{~Gb} / \mathrm{s}$ photodiodes (PDs). Next, the signals were sampled by a real-time digital signal oscilloscope (DSO) with sampling rate of $20 \mathrm{GS} / \mathrm{s}$ for offline digital signal processing (DSP) which mainly includes signal combining, hard decision and BER calculation. The three branches of received signals were combined using MRC algorithm with weight coefficients $\alpha_{\mathrm{i}}=P_{\mathrm{i}} / P$, where $P_{\mathrm{i}}$ is the power of the $i$ th branch of the received signals and can be expressed as $P_{i}=\left\|S_{i}\right\|^{2}$, where $S_{i}$ is the $i$ th branch of received signals and $P$ is the sum of power of the 3 branches of signals. The combined signals were then converted into binary symbols using hard decision with threshold $\Lambda=0.5 \times\left(I_{\max }+I_{\min }\right)$, where $I_{\max }$ and $I_{\min }$ represents the maximum and minimum amplitude of the combined signals respectively. Finally, the received signals were compared with the transmitted signals for BER calculations.

\section{EXPERIMENTAL RESULTS AND DISCUSSIONS}

The beam profiles of the output at collimator 1 (Col.1) and Col.2 and the beam splitter were captured using a charge coupled device (CCD) camera as shown in Fig.3, respectively. Overall, after combining the $\mathrm{LP}_{01}$ and the donut-shaped $\mathrm{LP}_{11}$ beam, the output beam has a good uniform intensity distribution despite a little lower (4.1\%) intensity in the center of the beam. The uniformity of the intensity profile of the resulting beam can be adjusted by optimising the power of the transmitted $\mathrm{LP}_{01}$ and $\mathrm{LP}_{11}$ beam and the pointing directions of Col.1 and Col.2.

Eye diagrams of the signals of 3 received modes and the combined signals are shown in Fig. 4 respectively when the received power at the lens system is $-11.7 \mathrm{dBm}$. It's clear that the combined signals performed significantly better than the signals of the 3 received modes. Among the received modes, $\mathrm{LP}_{01}$ has the best performance mainly because of its lower demultiplexing loss.

The BER performances of all three received modes separately and the combined signal are shown in Fig.5. It can be seen that the BER of the combining signals is lower than any other modes and $\mathrm{LP}_{01}$ has the lowest $\mathrm{BER}$ among the 3 received modes, which is consistent with the conclusions obtained from Fig.4. In addition, the combining signals have more obvious advantages at the region of low received optical power. Though there is a certain degree of performance difference between different modes and may introduce performance gaps among different users in the same cell, we believe that the performance difference and gaps can be further reduced when better mode demultiplexer with lower power loss can be used for experiments. Focus of this paper is to demonstrate the novel concept of using few modes to shape the beam and test the feasibility of this for indoor OWCs.
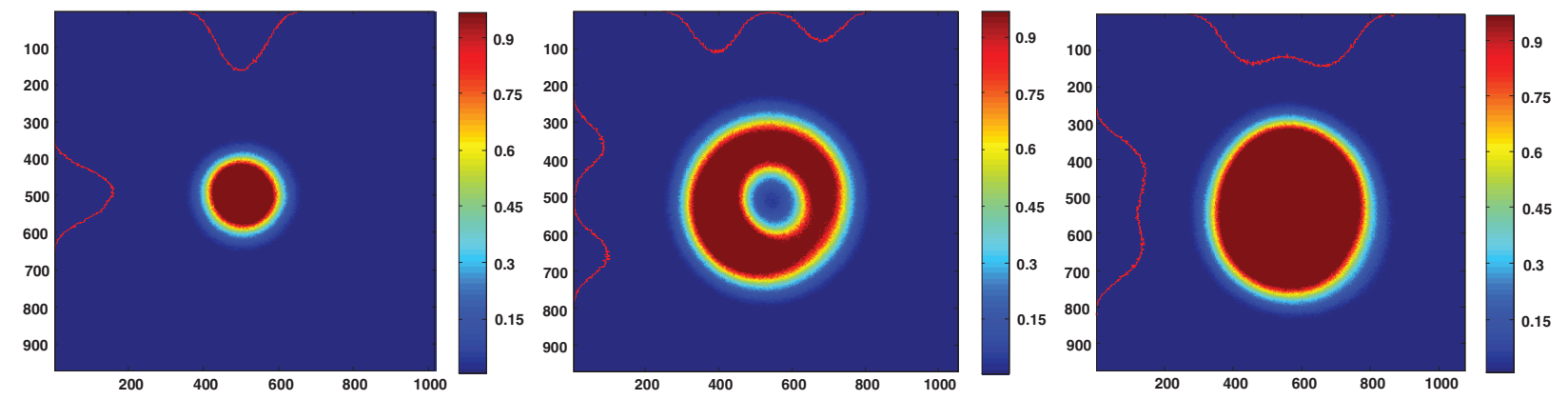

Fig. 3. $\mathrm{CCD}$ images of the intensity distribution of $\mathrm{LP}_{01}$ mode, donut-shaped $\mathrm{LP}_{11}$ mode and combined uniform beam. 

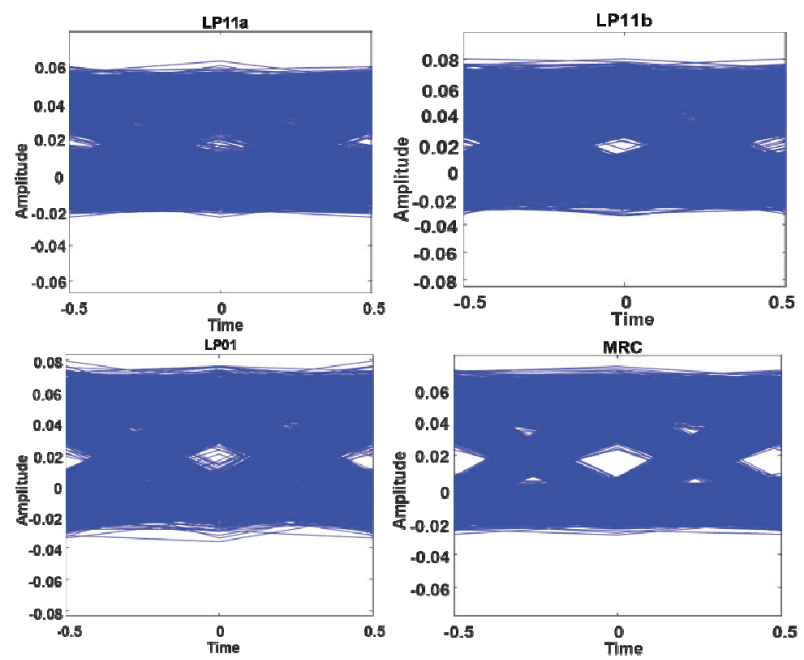

Fig. 4. Eye diagrams of the received signal.

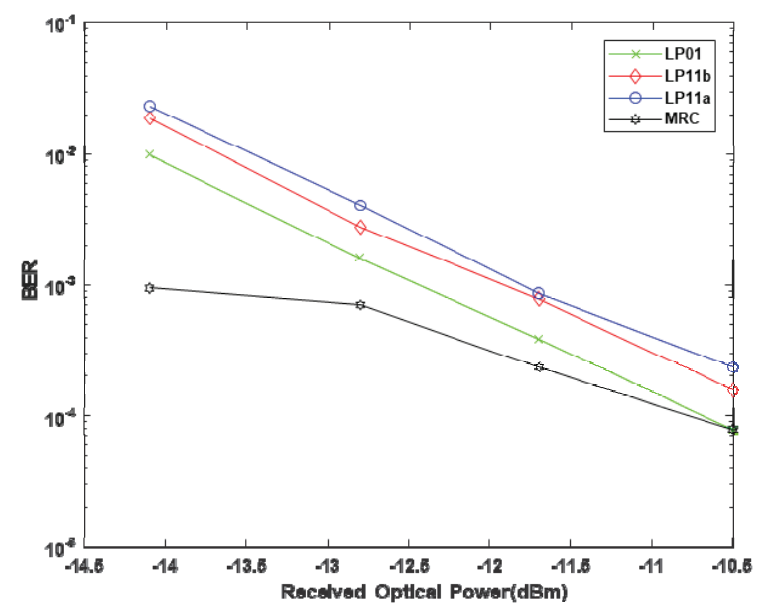

Fig. 5. BER performances.

\section{CONCLUSION}

In conclusion, in this paper, few-mode uniform beam shaping has been proposed for indoor OWCs to improve resistance to link outage and $10 \mathrm{~Gb} / \mathrm{s}$ transmission with a BER about $7.8 \times 10^{-5}$ has been achieved when received optical power is $-10.5 \mathrm{dBm}$. Furthermore, it has been shown that high-quality uniform beam with power variation below $4.1 \%$ can be attained. BER performance can be enhanced obviously using mode diversity reception and MRC algorithm.

\section{ACKNOWLEDGMENT}

The authors would like to thank Dr. Ranjith R. Unnithan from Department of Electrical and Electronic Engineering, The University of Melbourne for providing CCD camera for the experiments.

\section{REFERENCES}

[1] M. Z. Chowdhury, M. T. Hossan, A. Islam, and Y. M. Jang, "A comparative survey of optical wireless technologies: Architectures and applications," IEEE Access, vol. 6, pp. 9819-9840, 2018.
[2] A. Nirmalathas et al., "High-speed optical wireless communications for local area networks (invited)," in Proc. Opto-Electron. Commun. Conf., 2018, pp. 1-2.

[3] T. Song, A. Nirmalathas, C. Lim et al., "Performance Analysis of Repetition-Coding and Space-Time-Block-Coding as Transmitter Diversity Schemes for Indoor Optical Wireless Communications," J. Lightw. Technol., vol. 37, no. 20, pp. 5170-5177, Oct. 2019.

[4] J. A. Hoffnagle and C. M. Jefferson, "Design and performance of arefractive optical system that converts a Gaussian to a flattop beam," Appl. Opt., vol. 39, pp. 5488-5499, 2000

[5] D. M. Brown, F. M. Dickey, and L. W. Weichman, "Multi-aperture beam integration systems," in Laser Beam Shaping, F. M. Dickey and S. C. Holswade, Eds. New York: Marcel Dekker, 2000, p. 273.

[6] X. Gu, W. Mohammed, L. Qian, and P. W. E. Smith, "All-fiber laser beam shaping using a long-period grating," IEEE Photon. Technol. Lett. 20, pp. 1130-1132 (2008).

[7] W. Cheng, W. Han, and Q. Zhan, "Compact flattop laser beam shaper using vectorial vortex," Appl. Opt. 52, 4608 (2013).

[8] Safety of Laser Products, AS/NZS 2211.1: 2004, Mar. 2004. 


\section{University Library}

\section{- M M N E R VA A gateway to Melbourne's research publications}

Minerva Access is the Institutional Repository of The University of Melbourne

Author/s:

Li, J;Lim, C;Nirmalathas, A;O'Keefe, N;Lee, KL

Title:

Demonstration of High-speed Indoor Optical Wireless Communications using Few-mode Based Uniform Beam Shaping

Date:

2020-10-04

Citation:

Li, J., Lim, C., Nirmalathas, A., O'Keefe, N. \& Lee, K. L. (2020). Demonstration of Highspeed Indoor Optical Wireless Communications using Few-mode Based Uniform Beam Shaping. 25th Opto-Electronics and Communications Conference, OECC 2020, 00, IEEE. https://doi.org/10.1109/OECC48412.2020.9273565.

Persistent Link:

http://hdl.handle.net/11343/258810 